Dieses Dokument ist eine Zweitveröffentlichung (Postprint Version) / This is a self-archiving document (accepted version):

Adrian Ehrenhofer, Thomas Wallmersperger

A normalization concept for smart material actuation by the example of hydrogels

Erstveröffentlichung in / First published in:

PAMM: 89th Annual Meeting of the International Association of Applied mathematics and Mechanics, München, 2018. 2020. Wiley Online Library, Abschnitt 6. ISSN 1617-7061.

DOI: https://doi.org/10.1002/pamm.201800001

Diese Version ist verfügbar / This version is available on:

https://nbn-resolving.org/urn:nbn:de:bsz:14-qucosa2-741068 


\title{
A normalization concept for smart material actuation by the example of hydrogels
}

\author{
Adrian Ehrenhofer ${ }^{1, *}$ and Thomas Wallmersperger ${ }^{1, * *}$ \\ ${ }^{1}$ Institut für Festkörpermechanik, Technische Universität Dresden, George-Bähr-Str. 3c, 01069 Dresden \\ For passive (classical) materials, stress and strain are used to extract the material behavior from the sample behavior in a \\ tensile test. In analogy, the actuation behavior of active (smart) materials can be normalized. In the present research, we show \\ the normalization using the example of hydrogels that react with a volume change (swelling and deswelling) when exposed \\ to stimulus-changes like temperature, chemical concentrations, $\mathrm{pH}$ or light intensity changes. The normalized behavior can \\ then be implemented with the Temperature-Expansion-Model which is based on the analogy of active behavior with thermal \\ expansion. This allows the simulation of arbitrary active structures and the extraction of the sensitivity measure to a stimulus.
}

\section{Active behavior}

The actuation behavior of active (smart) materials is still very difficult t $\mathrm{og}$ rasp b y e ngineers $\mathrm{w}$ ho a re $\mathrm{n}$ ot $\mathrm{f}$ amiliar $\mathrm{w}$ ith the multi-physics backgrounds: These are for example electro-activity in Dielectric Elastomers, phase transitions in hydrogels, combined electro-chemical interactions in conductive polymers or phase transitions in shape memory alloys [1-3]. In the current work, we provide a concept based on the normalization and general representation of active behavior using the concept of analogies: Different phenomena can be described as analog if they can be represented by the same macroscopic model, i.e. mathematical representation. Well-known analogies are for example between electric and hydraulic circuits or between the thermal and chemical field. In the current work, we present how the active equilibrium behavior of different s mart materials can be described in analogy to thermal expansion.

\section{Description of the Temperature-Expansion-Model}

The approach of representing the isotropic swelling of the hydrogel poly(N-isopropylacrylamide) (PNiPAAm) with thermal expansion was called Temperature-Expansion-Model (TEM) $[4,5]$ in order to avoid confusion with thermal expansion which is a physical process. The TEM is a phenomenological approach to represent the active behavior using the same mathematical description, which is also done in various actuator equations.

Table 1: Equations for the Temperature-Expansion-Model (TEM), the extended (nonlinear) and the normalized version.

\begin{tabular}{llll}
\hline & TEM & Extended TEM & Normalized Extended TEM \\
Balance & $\sigma_{k l, k}+f_{l}=0$ & $\sigma_{k l, k}+f_{l}=0$ & $\sigma_{k l, k}+f_{l}=0$ \\
Kinematics & $\varepsilon_{k l}=\frac{1}{2}\left(u_{k, l}+u_{l, k}\right)$ & $\varepsilon_{k l}^{\mathrm{H}}=\frac{1}{2} \ln \left(F_{k M} F_{l M}\right)$ & $\varepsilon_{k l}^{\mathrm{H}}=\frac{1}{2} \ln \left(F_{k M} F_{l M}\right)$ \\
Mat. behavior & $\sigma_{k l}=E_{k l m n}\left(\varepsilon_{m n}-\alpha_{m n}^{\mathrm{th}} \Delta \vartheta\right)$ & $\sigma_{k l}=E_{k l m n}\left(\varepsilon_{m n}^{\mathrm{H}}-\beta_{m n}^{\mathrm{th}} \Delta \vartheta\right)$ & $\sigma_{k l}=E_{k l m n}\left(\varepsilon_{m n}^{\mathrm{H}}-\beta_{m n}^{\mathrm{F}} \Delta F^{\mathrm{Stimulus}}\right)$ \\
\hline
\end{tabular}

For small deformation gradients, thermal expansion can be obtained from basic engineering as HooKE's law i.e. the most basic material law from a first-order polynomial strain energy function [6], see Table 1 (left).

For finite deformations, different strain energy functions have to be found like e.g. the SAINT-VENANT-KIRCHHOFF or neo-HoOKE models [7]. A different approach is based on the multiplicative decomposition of the deformation gradient [8] $F_{k K}=F^{\text {th }} F_{k K}^{\mathrm{el}}$ into an isotropic strain for the volume expansion $F^{\text {th }}$ and an incompressible (isochoric) elastic part $F_{k K}^{\mathrm{el}}$. When the linear elasticity is described for the spatial logarithmic HENCKY-strain $\varepsilon_{k l}^{\mathrm{H}}=1 / 2 \ln \left(F_{k M} F_{l M}\right)$, the product inside the logarithm leads to an additive superposition of the strains in analogy to HoOKE's law. The nonlinear Extended TEM is given in Table 1 (middle).

The equations of the extended TEM can now be used with a thermal expansion coefficient $\beta_{m n}^{\text {th }}$ which is defined at a working point in the active behavior plot, see Figure 1. To implement arbitrary active material behavior, the actuation stimulus together with the expansion coefficient has to be described in a normalized way to account for non-temperature stimuli.

The tensorial form for the expansion coefficients allows the incorporation of possible direction dependencies in actuation like they can be found e.g. in Dielectric Elastomer Actuators. For isotropic expansion - as it can be observed in hydrogels or conductive polymers - they simplify to $\alpha_{k l}^{\text {th }}=\alpha^{\text {th }} \delta_{k l}$ and $\beta_{k l}^{\text {th }}=\beta^{\text {th }} \delta_{k l}$. 


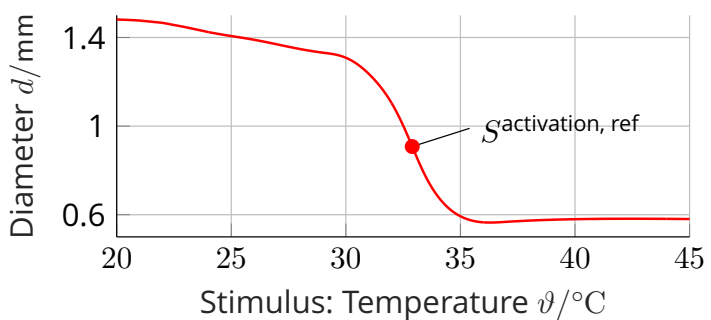

a)

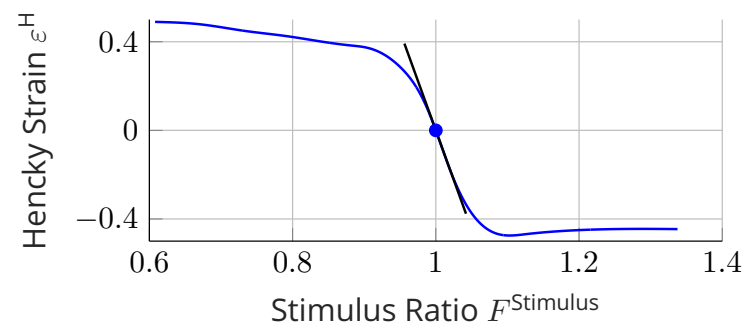

b)

Fig. 1: The diameter vs. stimulus curve denotes the physical swelling behavior $\mathbf{a}$. From this, the normalized behavior $\mathbf{b}$ can be derived.

Thermal expansion is usually implemented in commercial Finite-Element tools like Abaqus or Ansys. The initial boundary value problem composed of the momentum balance in reference configuration (LAGRANGEian description), kinematics (HENCKY strain) and linear elasticity can also be manually implemented and solved using e.g. Matlab.

\section{Normalization of hydrogel behavior}

The isotropic swelling of hydrogels can be understood in analogy to thermal expansion [4]. The activation stimulus $S^{\text {activation }}$ can be e.g. temperature $\vartheta, \mathrm{pH}$ or chemical concentration. As a replacement for $\vartheta$ in the material law, the stimulus ratio $F^{\text {Stimulus }}=S^{\text {activation }} / S^{\text {activation, ref }}$ can be defined. This normalizes the stimulus, i.e. it removes the dimensions and transforms stimuli to a similar range e.g. $(0, \ldots, 10)$. The reference stimulus $S^{\text {activation, ref }}$ can be found at a characteristic point of the stimulus curve, e.g. the inflexion point in Figure 1.

In a very limited stimulus range, the actuation response to a stimulus can be linear, see Figure 1b. Then, a linear expansion coefficient $\alpha^{\text {th }}$ can be found and a parameter $\mathcal{D}^{\gamma}$ - called sensitivity in analogy to signal processing - which depicts a mechanical compliance to an outer stimulus $\gamma$ (e.g. temperature, $\mathrm{pH}$ ), can be derived. This parameter can be understood as an inverse elastic modulus in a stress-strain relation. Different active materials reacting to the same stimulus can be compared in their sensitivity measure just as passive materials are compared in their elastic modulus.

For any nonlinear expansion behavior over the stimulus, a nonlinear and stimulus-dependent expansion coefficient $\beta\left(S^{\text {activation }}\right)$ can be derived. This can be achieved through fitting of the free actuation, e.g. for hydrogels free swelling. If only data for the constrained form is available, the variational problem consisting of the balance laws, kinematics and constitutive equations with the target function $\beta^{\mathrm{F}}\left(F^{\text {Stimulus }}\right)$ can be formulated to obtain the expansion coefficient.

\section{Conclusion and Outlook}

The Normalized Extended Temperature-Expansion-Model provides an easy tool to grasp the active behavior of smart materials. It is based on the phenomenological approach for the active behavior and represents this by the analogy to thermal expansion, which is usually well implemented in commercial Finite-Element tools. Through the normalization concept, arbitrary activation stimuli can be incorporated even when they are nonlinear. The approach can further be used in other engineering problems that consider thermal expansion e.g. inside the framework of Classical Laminate Plate Theory for composites.

As for any material description on the continuum level with constitutive laws, the Temperature-Expansion-Model has several limitations like e.g. the neglect of involved transport processes. The model can be further expended to include these effects which makes the model more accurate in the phenomenological description of the active behavior. At the same time, the model will become more complex and harder in implementation. In future works, we will further explore the limitations of the model. Inside the provided boundaries, the Temperature-Expansion-Model can be used as a tool for the description of active materials without further insight into the underlying mechanisms. This can make design and construction of active structures much more accepted in industry and can therefore be a step to foster the advance of active structures.

\section{References}

[1] Y. Bar-Cohen, in: Electroactive Polymers as Artificial Muscles: Capabilities, Potentials and Challenges (American Society of Civil Engineers, 2000), chap. 23, $188-196$.

[2] T. F. Otero and J. G. Martínez, in: Electromechanically Active Polymers, edited by F. Carpi (Springer, 2016), chap. Conducting Polymers as EAPs: Fundamentals and Materials, $237-255$.

[3] G. Gerlach and K. F. Arndt, Hydrogel Sensors and Actuators: Engineering and Technology (Springer, 2009).

[4] A. Ehrenhofer, M. Elstner, and T. Wallmersperger, Sensors and Actuators B: Chemical 255(2), 1343 - 1353 (2018).

[5] A. Ehrenhofer, G. Bingel, G. Paschew, M. Tietze, R. Schröder, A. Richter, and T. Wallmersperger, Sensors and Actuators B: Chemical 232, $499-505$ (2016).

[6] H. Altenbach, Kontinuumsmechanik: Einführung in die materialunabhängigen und materialabhängigen Gleichungen (Springer, 2015).

[7] G. A. Holzapfel, Nonlinear Solid Mechanics: A Continuum Approach for Engineering (John Wiley \& Sons, Inc., 2000).

[8] S. Galante, A. Lucantonio, and P. Nardinocchi, International Journal of Non-Linear Mechanics 51, 112 - 120 (2013). 